\title{
Protocol for a multicenter prospective cohort study evaluating sport activity and development of femoroacetabular impingement in the adolescent hip
}

\author{
Axel Öhlin, Nicole Simunovic, Andrew Duong, Olufemi R. Ayeni ${ }^{*}$ and PREVIEW investigators
}

\begin{abstract}
Background: Femoroacetabular impingement (FAl) is an important cause of hip pain in young and active individuals and occurs as a result of size and shape mismatch between the femoral head and acetabulum. Open physes in children can make hips more susceptible to injury, and high impact forces have been suggested to affect the developing femur. The diagnosis of FAl has recently risen, especially within adolescent populations, and there is an increasing trend towards yearround participation in sports with early specialization. The PREVIEW study is an international longitudinal study designed to determine the association between sport specialization in adolescence and the development of hip impingement.
\end{abstract}

Methods: This is a multicentre prospective cohort study evaluating 200 participants between the ages of 12-14 that include sport specialists at the moderate to vigorous physical activity (MVPA) level and non-sport specialists at any activity level. We will monitor physical activity levels of all participants using an activity log and a wrist-mounted activity tracker, with synced data collected every 3 months during the study period. In addition, participants will be evaluated clinically at 6, 12, and 18 months and radiographically at the time of enrolment and 24 months. The primary outcome is the incidence of FAl between groups at 2 years, determined via MRI. Secondary outcomes include hip function and health-related quality of life between subjects diagnosed with FAl versus no FAl at 2 years, as determined by the Hip Outcome Score (HOS) and Pediatric Quality of Life (PedsQL) questionnaires.

Discussion: It is important to mitigate the risk of developing hip deformities at a young age. Our proposed prospective evaluation of the impact of sport activity and hip development is relevant in this era of early sport specialization in youth. Improving the understanding between sport specialization and the development of pre-arthritic hip disease such as FAl can lead to the development of training protocols that protect the millions of adolescents involved in sports annually.

Trial registration: PREVIEW is registered with clinicaltrials.gov (NCT03891563).

Keywords: Femoroacetabular impingement, Magnetic resonance imaging, Sport specialization, Adolescents

\footnotetext{
* Correspondence: ayenif@mcmaster.ca

Department of Surgery, Division of Orthopaedic Surgery, McMaster

University, 1200 Main St West, 4E15, Hamilton, ON L8N 3Z5, Canada

(c) The Author(s). 2020 Open Access This article is licensed under a Creative Commons Attribution 4.0 International License, which permits use, sharing, adaptation, distribution and reproduction in any medium or format, as long as you give appropriate credit to the original author(s) and the source, provide a link to the Creative Commons licence, and indicate if changes were made. The images or other third party material in this article are included in the article's Creative Commons licence, unless indicated otherwise in a credit line to the material. If material is not included in the article's Creative Commons licence and your intended use is not permitted by statutory regulation or exceeds the permitted use, you will need to obtain permission directly from the copyright holder. To view a copy of this licence, visit http://creativecommons.org/licenses/by/4.0/. The Creative Commons Public Domain Dedication waiver (http://creativecommons.org/publicdomain/zero/1.0/) applies to the data made available in this article, unless otherwise stated in a credit line to the data.
} 


\section{Background}

Femoroacetabular impingement (FAI) can cause hip pain and may lead to the development of osteoarthritis of the hip later in life. Some cross-sectional studies have estimated that the prevalence of hip impingement ranges from 14 to $17 \%$ among asymptomatic young adults to almost 95\% among competitive athletes [1-3]. FAI occurs as a result of a size and shape mismatch between the femoral head and the acetabulum. FAI is typically classified into 2 subtypes; cam-type (a misshaped femoral head) or pincer type (an over covered or deep socket). Most adult patients (18+ years) have a combination of both types of impingement [4]. With FAI, the abnormal femoral head and acetabular rim of the hip joint collide or "impinge" during movements such as hip flexion and rotation [5]. Typically, patients with this condition experience hip pain and loss of hip function. The development of hip pain in this manner serves as an indicator for early cartilage and labral damage, potentially resulting in hip osteoarthritis [5].

The incidence of FAI has recently risen across all age groups, but it has been especially notable within paediatric, or more specifically, adolescent populations [6]. In the adult, FAI is most commonly attributed to an "idiopathic anatomic variant" [6]. In the paediatric population, implicated causes of FAI have included genetics, subclinical paediatric hip disease, and stresses applied to the hip joint from certain high-intensity sports [7]. According to Packer et al., there is no definitive evidence that FAI is transmitted genetically, and in otherwise healthy children, there is growing evidence that FAI has a higher prevalence in athletes who performed at a high level during adolescence [8-11].

High impact activities in combination with intensity of various kinds have been shown to affect the developing femur [12]. Among children, open physes and growing cartilage make them more susceptible to injury and shear forces that can result in premature physeal arrest, apophyseal avulsion fractures, and chondral injuries [13]. A higher prevalence of cam deformities (> 50\%), both symptomatic and asymptomatic, has been shown in adolescent athletes that play ice hockey, basketball, and soccer when compared to controls that did not play sports [7, 10, 11, 14-17]. These sports involve repetitive deep flexion, flexion-adduction or extension-abduction movements, which bring the cam lesion on the femoral head or the pincer lesion on the acetabulum into conflict [6]. Therefore, participating in high impact sports during growth likely plays an important role in the development of hip deformities. This is concerning given: a) the increasing trend toward year-round participation in youth sports with early specialization and b) FAI has been shown to predispose patients to the progression of hip osteoarthritis [18-21]. Still, most studies in the current literature that evaluate the relationship between sports and the development of FAI are relatively small, retrospective case-controls and the most recent evidence demonstrates conflicting results regarding how and when primary FAI develops in relation to skeletal maturity [7, 22]. Accordingly, the current literature notes the need for longitudinal or prospective magnetic resonance imaging (MRI) studies to understand the etiology of primary FAI development to identify preventive strategies, delineate radiographic values, define specific indications for operative management, and examine long-term outcomes to determine optimal management $[7,12]$.

\section{Study objectives}

The primary research objective is to determine if sport specialization at the moderate-to-vigorous physical activity (MVPA) level [23-27] is associated with the development of both symptomatic and asymptomatic FAI (i.e. cam, pincer, or mixed-type) in adolescents (ages of 12-14 years) at 2 years post-enrollment determined via MRI.

The secondary research objectives are to determine if the presence of FAI is associated with changes in hip function and health-related quality of life (HRQL) at 2 years post-enrollment determined via the Hip Outcome Score (HOS) and Pediatric Quality of Life (PedsQL) questionnaires.

\section{Methods \\ Study design}

This is a longitudinal cohort study of 200 participants between the ages of 12 and 14 (i.e. critical age of development of the femoral head and neck). Participants will be evaluated clinically at enrollment and 6, 12, 18, and 24 months and radiographically (with MRI) at baseline and 24 months. Activity will be tracked every 3 months from enrollment to final follow-up at 2 years. Participants will be recruited from experienced hip surgeons and sports medicine researchers at multiple international clinical sites. We will observe the incidence of radiographic FAI, as determined by MRI of the hip, and evaluate hip function and HRQL using the HOS and PedsQL, respectively. Ethics approval for this study was granted by the Hamilton Integrated Research Ethics Board (Version D1.0, 1-August-2019, HIREB \#7829). Any protocol amendments will be submitted to HIREB and communicated to all participating sites for submission their site-specific ethics boards.

\section{Participant selection Eligibility criteria}

The inclusion criteria are: 1) asymptomatic males and females between the ages of 12-14 years; 2) ability to speak, understand, and read the language of the clinical site; and 3) provision of informed child assent (if applicable) and parental consent. 
The exclusion criteria mainly preclude known confounding factors that would impede the evaluation of the etiology of a hip deformity. These include: 1) hip is mature (i.e. closed physes) based on the baseline MRI scan; 2) hip complaints or pain in the hip; 3) previous trauma to the hip; 4) previous surgery on the hip; 5) significant medical co-morbidities (requiring daily assistance for activities of daily living); 6) history of or ongoing paediatric hip disease (Legg-Calve-Perthes; slipped capital femoral epiphysis, hip dysplasia); 7) participants that have contraindications and/ or are unwilling to undergo an MRI (e.g. claustrophobia); 8) participants who will likely have problems, in the judgment of the investigator, with maintaining follow-up; and 9) any other reasons the investigator feels is relevant for excluding the subject (e.g. participant is planning to move in the next 2 years, unreliable access to transportation to attend follow-up visits, etc.).

\section{Participant recruitment and screening}

The study activities at each participating clinical site will be led by experienced hip surgeons and sports medicine physician researchers. However, because we are looking for healthy volunteers, participants might not be exclusively recruited from the co-investigators' clinics. Instead, we will use several recruitment strategies that have proven successful in our pilot study. These include: 1) posting study flyers throughout each participating institution; 2) distributing study flyers to grades in the eligible age range at school boards in the regions of the recruiting institutions; and 3) having the co-investigators reach out to community coaches and competitive sport organizations and clubs, and presenting the study to the organizers, parents, and adolescent athletes. Potentially eligible subjects will be approached along with their parents/ guardians for consent for participation.

\section{Study exposure}

The study groups will be defined by 'exposure' to varying levels of physical activity (i.e. exposure variables) and include sport specialists at the MVPA level (group 1) and non-sport specialists at any activity level (group 2).

\section{Defining sport specialization}

Sport specialization will be defined according to the American Orthopaedic Society for Sports Medicine (AOSSM) and American Medical Society for Sports Medicine (AMSSM) early sport specialization criteria (Table 1 ) [28, 33]. At the baseline/enrollment visit, we will collect information about any current sport participation, including type(s) and frequency, for each participant. One week prior to each followup visit, we will ask participants and their parent(s) to complete a daily activity log to record all types of sport activity the participant engaged in (e.g. games, practices, both organized and unorganized), or lack thereof.

\section{Defining activity level}

We will cross-reference the activity log with data from an objective wrist-worn activity tracker (Garmin ${ }^{\bullet}$ Vivofit 4) to define sport and other activity intensity [34]. The Vivofit is a small, lightweight, waterproof activity tracker with high validity (relative to reference measures of physical activity and direct observation), high reliability, low reactivity in children, and has been used successfully in multiple paediatric physical activity studies [26, 3537]. The Vivofit utilizes accelerometer measurements to calculate the Metabolic EquivalenTs (METs) of energy expenditure, where activity greater than 3 METs corresponds to MVPA. The categorical activity output includes data for sedentary, fairly active, and MVPA levels. Relevant data also includes duration of MVPA and number of total active minutes per day [38]. We will use the cut-off of $\geq 180$ min [31,32] of MVPA per week detected by the tracker during recorded sport participation (as

Table 1 Definition of study groups according to objective measurement and guideline criteria

\begin{tabular}{|c|c|c|c|}
\hline Study group & AOSSM criteria [28] & Activity tracker criteria & Duration \\
\hline $\begin{array}{l}\text { Group 1: Sport } \\
\text { specialist, MVPA }\end{array}$ & $\begin{array}{l}\text { 1. Participation in intensive training and/or } \\
\text { competition in organized sports greater than } 8 \\
\text { months per year (essentially year round) [29] } \\
\text { 2. Participation in } 1 \text { sport to the exclusion of } \\
\text { participation in other sports (limited free play } \\
\text { overall) [30] } \\
\text { 3. Involving prepubertal (seventh grade or roughly } \\
\text { age } 12 \text { years) children. }\end{array}$ & $\begin{array}{l}\text { Greater than } 180 \text { accumulated minutes of MVPA } \\
{[31,32] \text { during participation in one sport type }} \\
\text { across one week of activity tracking }\end{array}$ & $\begin{array}{l}\text { Meets criteria within } \\
\text { either one or both } \\
\text { years of follow-up }\end{array}$ \\
\hline $\begin{array}{l}\text { Group 2: Non- } \\
\text { sport specialist, } \\
\text { any activity level }\end{array}$ & $\begin{array}{l}\text { 1. Participation in more than } 1 \text { sport at any } \\
\text { physical activity level } \\
\text { OR } \\
\text { 2. Participation in none or low training and/or } \\
\text { competition in organized sports for any period of } \\
\text { time. } \\
\text { 3. Involving prepubertal children. }\end{array}$ & $\begin{array}{l}\text { Greater than or less than } 180 \text { accumulated } \\
\text { minutes of MVPA across one week of activity } \\
\text { tracking }\end{array}$ & $\begin{array}{l}\text { Meets criteria during } \\
\text { both years of follow- } \\
\text { up }\end{array}$ \\
\hline
\end{tabular}


per the activity log) to define the MVPA level in group 1 (Table 1). The use of the activity tracker, in conjunction with self-reported activity logs has been shown to be accurate in estimating energy expenditure in adolescents $[39,40]$.

One week following the baseline visit and 1 week prior to each follow-up visit, participants will wear the tracker on their wrist for a full week and to sync it with a paired tablet or cellular phone. Measuring total 'volume' physical activity at several time points by one-week intervals is a standard methodology used in several paediatric physical activity studies [25-27]. The combination of the activity measures and criteria described above will be used to define the study groups as per Table 1 . The study groups will be defined at the completion of follow-up given participant activity levels may change over time.

\section{Study outcomes}

\section{Primary outcome}

The primary outcome is the incidence of radiographic FAI between groups at 2 years, as determined by the dedicated MRI of the hip (Table 2). We will identify both symptomatic and asymptomatic cases, where asymptomatic FAI can become symptomatic in young adulthood, and/or could be associated with idiopathic osteoarthritis later in life [41].

\section{Secondary outcomes}

Secondary outcomes include: 1) hip function and 2) HRQL between subjects with and without any type of FAI at 2 years, as determined by the HOS and PedsQL questionnaires $[42,43]$.

\section{Outcome measures}

Participants will complete the study questionnaires and undergo a physical examination (at baseline and 6, 12, 18 , and 24 months) and MRI (at baseline and 24 months) of their dominant hip. Participants will sync their activity tracker data and send their activity log (at baseline and every 3 months until final follow-up at 2 years) (Table 3 ).

Table 2 Criteria for defining FAl types

\begin{tabular}{|c|c|}
\hline $\begin{array}{l}\text { FAl } \\
\text { type }\end{array}$ & Criteria \\
\hline Cam & $\begin{array}{l}\text { 1. Alpha angle is }>55^{\circ} \text {; } \\
\text { 2. Anterior head-neck offset }<10 \mathrm{~mm} \text {; } \\
\text { 3. Anterior head-neck offset ratio }<0.15 \\
{ }^{*} \text { Must meet } 2 / 3 \text { criteria to be considered cam impingement }\end{array}$ \\
\hline Pincer & $\begin{array}{l}\text { 1. Acetabular depth }<3 \mathrm{~mm} \text {; } \\
\text { 2. Lateral centre-edge angle }>39^{\circ} \text {; } \\
\text { 3. Acetabular anteversion (in the upper third of the femoral } \\
\text { head) }<0^{\circ} \\
{ }^{*} \text { Must meet } 2 / 3 \text { criteria to be considered pincer impingement }\end{array}$ \\
\hline Mixed & $\begin{array}{l}\text { *Must meet 2/3 criteria each for cam and pincer impingement } \\
\text { (as per above) to be considered mixed impingement }\end{array}$ \\
\hline
\end{tabular}

FAl Femoroacetabular impingement
The physical examination will include range of motion (ROM) measurements in all planes documented in the supine and prone positions by a blinded research assistant (using a goniometer), as well as response of provocative hip tests on both hips [44]. Symptomatic FAI in adolescents is typically characterized by anterior hip pain aggravated by flexion activities, decreased hip internal rotation, and a positive impingement sign [7]. We will define symptomatic FAI using the following criteria: 1 ) positive impingement with groin pain based on flexion, adduction, and internal rotation (FADIR) test, and 2) decreased ROM: internal rotation of the hip when compared to the contralateral side by 5 degrees $[45,46]$.

We will use a non-contrast three dimensional (3D)volumetric interpolated breath-hold examination (VIBE) sequence MRI [47]. This protocol has been used to document FAI-related morphology and eliminates the use of intra-articular contrast injections. All MRI and physical examination information will be provided to an independent radiologist adjudicator to determine the incidence of cam, pincer, or mixed-type FAI according to the pre-specified criteria (Table 2). We will also document the change in cartilage volume, labral pathology, herniation pits on the femoral neck, and the extent of physeal closure as these variables may predict future hip osteoarthritis [44].

The HOS is a self-administered questionnaire designed to capture hip function with clinimetric evidence for use in patients with FAI or labral tears [34, 36, 37]. The HOS was developed for young adults and has been shown to be one of the only hip function questionnaires appropriate for use in adolescents [36, 37, 48]. The PedsQL is a validated and responsive measure of HRQL in children [43]. The PedsQL was specifically designed to measure the core health dimensions outlined by the World Health Organization (physical, emotional, and social functioning), which will ensure generalizability across our global sites [49]. Adult and child versions of the HOS and PedsQL will be completed by a parent and participant at each visit. Adverse events, defined as any symptom, sign, illness, or experience that develops or worsens in severity during the course of this study, will also be documented (Table 3).

\section{Study follow-up}

We will follow participants for 2 years as this timeframe is expected to coincide with the critical period of physeal closure in our study population. In-person follow-up visits will occur at baseline (i.e. time of enrollment), and at $6,12,18$, and 24 months post-enrollment. Leading up to each follow-up visit, we will ask each participant to wear their activity tracker for 1 week and maintain a daily activity log. At the follow-up visit, we will collect the activity data collected in the week prior, perform a 
Table 3 Schedule of events for the PREVIEW study

\begin{tabular}{|c|c|c|c|c|c|c|c|c|c|}
\hline Data collection & Enrollment & $3 \mathrm{M}$ & $6 \mathrm{M}$ & $9 M$ & $12 \mathrm{M}$ & $15 \mathrm{M}$ & $18 \mathrm{M}$ & $21 \mathrm{M}$ & $24 \mathrm{M}$ \\
\hline Screening and informed consent & $\bullet$ & & & & & & & & \\
\hline Enrolment data (Demographics) & $\bullet$ & & & & & & & & \\
\hline Physical hip examination & $\bullet$ & & $\bullet$ & & $\bullet$ & & $\bullet$ & & $\bullet$ \\
\hline MRI & $\bullet$ & & & & & & & & $\bullet$ \\
\hline Follow-up form & $\bullet$ & & $\bullet$ & & $\bullet$ & & $\bullet$ & & $\bullet$ \\
\hline Activity tracker wear \& synchronization & * & $x$ & $x$ & $x$ & $x$ & $x$ & $x$ & $x$ & $x$ \\
\hline Activity log & * & $x$ & $x$ & $x$ & $x$ & $x$ & $x$ & $x$ & $x$ \\
\hline Hip Outcome Score (HOS) & $\bullet$ & & $\bullet$ & & $\bullet$ & & - & & $\bullet$ \\
\hline Pediatric QoL Inventory (PedsQL) & $\bullet$ & & $\bullet$ & & $\bullet$ & & $\bullet$ & & $\bullet$ \\
\hline Adverse events & & & $\bullet$ & & $\bullet$ & & $\bullet$ & & $\bullet$ \\
\hline
\end{tabular}

M months, MRI Magnetic Resonance Imaging, ${ }^{*}=1$-week post-enrollment/baseline visit, $\mathrm{x}=1$-week prior to visit

physical hip examination, and ask participants to complete the function and HRQL questionnaires. We will have 4 additional remote activity monitoring visits at 3, 9, 15, and 21 months. Each participant will undergo an MRI of their dominant hip at the baseline and 24month visits. We designed the follow-up schedule to track activity every 3 months, maintain regular contact with the participants to help minimize loss to follow-up, and at the same time, allow enough time between inperson visits to minimize participant burden.

\section{Sample size calculation}

We have based our sample size calculation on our pilot study data $(N=53)$, where the primary outcome event rate (incidence of FAI) is $40 \%$ for the sport specialists at the MVPA level (group 1) and 15\% for the non-sport specialists at all physical activity levels (group 2). These proportions very closely match prior cross-sectional research that evaluated the incidence of FAI among athletes [10]. Because we have looked at early data from the pilot study to inform our calculation, we have adjusted our type I error rate. To achieve $80 \%$ power for a two-sided test at alpha $=\underline{0.01}$, we will require a sample size of 81 in each exposure group (Table 4). Given that some participants' activity levels may change during the course of the study (where they may change from sport specialist to non-specialist, or vice versa), we will increase the sample size by $10 \%$ to ensure we have at least 81 participants in each exposure group. We will also account for a $5 \%$ rate of ineligibility (due to a mature hip [closed physes] found on the baseline MRI) and a $10 \%$ loss to follow-up rate. Therefore, we require a total sample size of 200 participants. The pilot participants will be rolled into the definitive study.

\section{Statistical plan}

\section{Primary analysis}

As previously described, study groups will be defined at the completion of follow-up given participant activity levels may change over time. The proportion of subjects developing FAI in the sport specialist group versus nonsport specialist group will be compared using a logistic regression analysis with FAI as the dependent variable and will include the following independent variables: activity level (sport specialist versus non-sport specialist), body mass index (BMI), and sex. Results will be reported as odds ratios (OR) with $95 \%$ confidence intervals and associated $p$-values.

\section{Secondary analyses}

We will perform independent samples t-tests to test for differences in 24-month HOS and PedsQL scores between participants who do and do not develop FAI within the 24-month follow-up period. These analyses will be repeated comparing participants who do and do not develop symptomatic FAI. Each secondary outcome will be quantified using descriptive statistics and 95\% confidence intervals. We will use multiple imputation to handle missing data.

\section{Subgroup/sensitivity analyses}

To assess whether the magnitude of the effect of sport activity on the development of FAI is significantly different in males than in females, we will add an interaction term between sex and sport specialist versus non-sport specialist to the primary logistic regression model. We

Table 4 Per group sample sizes. 80\% power, alpha =0.01, 2-sided testing

\begin{tabular}{cccc}
\hline \multicolumn{4}{c}{ FAl risk in non-sports specialists } \\
\cline { 2 - 4 } & $5 \%$ & $15 \%$ & $25 \%$ \\
\hline FAI risk in sports specialists & & 1668 \\
$20 \%$ & 126 & 1388 & 1902 \\
$30 \%$ & 61 & 193 & 240 \\
$40 \%$ & 38 & 81 & 94 \\
$50 \%$ & 26 & 46 & 94 \\
\hline
\end{tabular}


hypothesize that sport specialization will be more strongly associated with incidence of FAI in males than in females.

No interim analyses are planned. All tests will be 2sided with $\alpha=0.01$. We will use SAS 9.4 (Cary, NC) to perform all analyses.

\section{Study committees and monitoring}

The Steering Committee will provide guidance and direction to the overall study. Specific responsibilities of the Steering Committee include reviewing and approving the study protocol and working together to resolve any challenges that arise during the study. The Steering Committee will also be supported by several Advisory Committees including: radiology, orthopaedic surgery, physiotherapy/training, and paediatrics and rehabilitation medicine. These Advisory Committees consist of individuals who are experts within their fields and have committed time to provide advice to the Steering Committee throughout the initiation and conduct of this study. An independent adjudication committee blinded to each participant's activity level, will determine the incidence of cam, pincer, or mixed-type FAI (primary outcome) and evaluate all adverse events reported during the study. Ongoing remote and in-person clinical site monitoring will be performed by experienced Methods Centre personnel (Fig. 1).

\section{Data management}

The case report forms (CRFs) will be the primary data collection tool for the study. All data requested on the CRF must be recorded. An Electronic Data Capture (EDC) system (iDataFax) will be used to submit data to the Methods Centre located at McMaster University. Upon receipt of the data, the personnel at the Methods Centre will make a visual check of the data and they will query all missing data, implausible data, and inconsistencies.

\section{Ethics and dissemination}

Any subjects who meet all eligibility criteria should be approached to discuss participation in the study by someone on the study team who is knowledgeable about the study. Study personnel must approach the subject and their parent(s)/legal guardian about the study in accordance with ethical requirements for consenting children into research studies. The study protocol, clinical site-specific informed consent forms, and any participant recruitment material will need to be reviewed and approved by each clinical site's local Ethics Board.

Information about study participants will be kept confidential and stored securely at each clinical site, only accessible to study personnel. Study records will be identified only by a coded participant number, and all records that contain participants' names or other identifying information will be stored separately. All local databases used for storage of study data will be password protected.

\section{Discussion}

The rationale for the PREVIEW study includes: 1) The number and diagnoses of FAI has recently risen and is especially notable within the adolescent populations; 2 ) High impact and high intensity activity common in many sports have the potential to cause hip damage, especially during physeal closure in the young; 3 ) There is an increasing trend toward year-round participation in youth sports with early specialization; and 4) An uncertainty regarding "how much is too much" of the same sport activity.

The PREVIEW study is one of the largest prospective cohort studies using MRI to determine the relationship between FAI development during skeletal maturation and physical activity. The eligibility criteria and followup period will help to ensure we evaluate the effect of sport activity during the critical phase of hip development and maturation. The sample size calculation is based on actual pilot data to ensure statistical power to detect differences in the incidence of FAI, hip function and HRQL among groups. Outcome assessment bias will be minimized by the use of an objective assessment tool for physical activity as well as independent adjudication of the primary outcome. The feasibility of the study has been demonstrated by the successful completion of the pilot study enrollment.

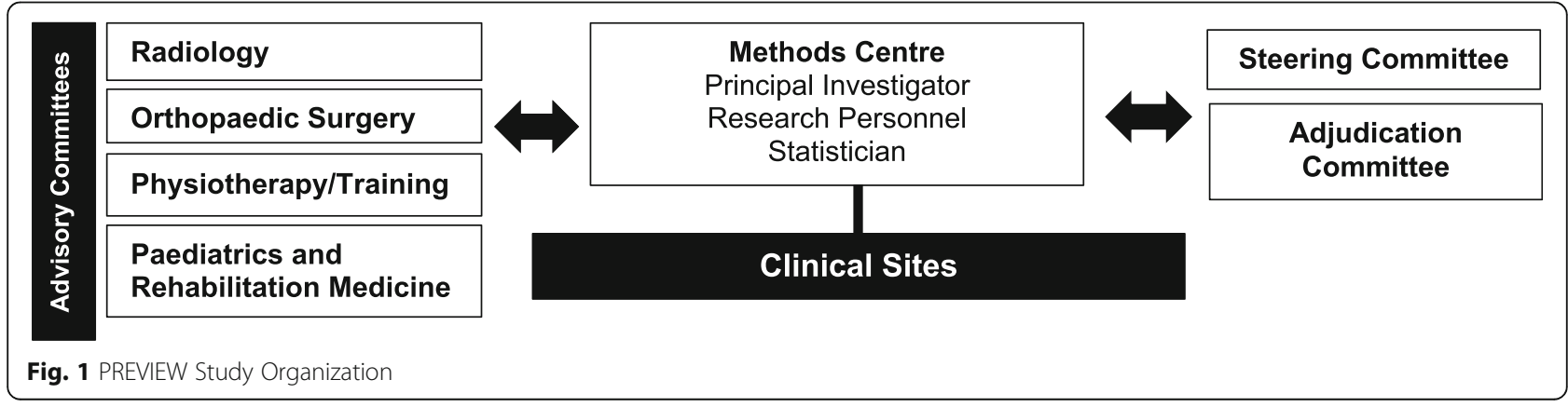


As FAI is diagnosed most frequently in athletes, and it is estimated that 30 to 45 million children and adolescents age 6-18 years old are involved in sports in the US alone, it is becoming imperative to identify factors that may predict its development, study treatments, and improve outcomes. Considering that research has demonstrated a relationship between FAI and osteoarthritis in adulthood, potentially leading to the need for total hip replacement, it has now become critical to mitigate the risk of developing these hip deformities at a young age [6]. Within organized sports, there is a current trend among coaches and parents to have children focus on one sport from a young age (i.e. sport specialization), with the thought that this dedication will allow them to reach an elite level $[28,33]$. If sport specialization is associated with the development of FAI in adolescents, then there is an important opportunity to evaluate and implement improved screening and prevention strategies as well as early intervention options. A prospective evaluation of the impact of sport specialization across all types of sport during the period of hip maturation is needed in order to identify, and subsequently protect, the thousands of young people that may be at risk of developing this condition.

\section{Abbreviations \\ 3D: Three dimensional; AMSSM: American Medical Society for Sports Medicine; AOSSM: American Orthopaedic Society for Sports Medicine; BMI: Body Mass Index; CRF: Case Report Form; EDC: Electronic Data Capture; FADIR: Flexion, ADduction, and Internal Rotation; FAl: Femoroacetabular Impingment; HOS: Hip Outcome Score; HRQL: Health-Related Quality of Life; METs: Metabolic EquivalenTs; MID: Minimally Important Difference; MRI: Magnetic Resonance Imaging; MVPA: Moderate-to-Vigorous Physical Activity; OR: Odds Ratios; PedsQL: Pediatric Quality of Life; PREVIEW: The Prospective Evaluation of Sport Activity and the Development of Femoroacetabular Impingement in the Adolescent Hip; ROM: Range Of Motion; SD: Standard Deviation; VIBE: Volumetric Interpolated Breath-hold Examination}

\section{Acknowledgements}

PREVIEW Investigators Authorship:

McMaster University (Canada) - Olufemi R. Ayeni, Nicole Simunovic, Andrew Duong, Yan Sim, Lehana Thabane, Matthew Skelly, Ajay Shanmugaraj, Diane Heels-Ansdell, Lisa Buckingham.

University of Pittsburgh (USA) - Volker Musahl.

Hospital da Luz (Portugal) - Vasco V. Mascarenhas.

CHU de Québec-Université Laval (Canada) - Etienne L. Belzile, Sylvie Turmel. Erasmus University Medical Centre Rotterdam (The Netherlands) - Rintje Agricola.

Kyungpook National Hospital (South Korea) - Seung-Hoon Baek.

University of Gothenburg (Sweden) - Axel Öhlin.

\section{Authors' contributions}

OA and NS and all PREVIEW Investigators listed in the authorship appendix made substantial contributions to conception and design, or acquisition of data, or analysis and interpretation of data. AO, NS, AD, OA, and the Steering Committee members (VM, YS, LT) have been involved in drafting the manuscript or revising it critically for important intellectual content. All authors read and approved the final manuscript.

\section{Author's information}

Writing Committee: Axel Öhlin MD (University of Gothenburg), Nicole Simunovic MSc (McMaster University), Andrew Duong MSc (McMaster University), Olufemi R. Ayeni MD, MSc, PhD, FRCSC (McMaster University).
Steering Committee: Olufemi R. Ayeni MD, PhD, FRCSC (Chair, McMaster University), Volker Musahl MD (University of Pittsburgh), Yan Sim MD (McMaster University), Lehana Thabane PhD (McMaster University). Methods Centre: Olufemi R. Ayeni MD, PhD, FRCSC (Principal Investigator); Nicole Simunovic MSc (Research Manager); Andrew Duong MSc, Matthew Skelly MSc, Ajay Shanmugaraj BSc (Project Management); Diane HeelsAnsdell MSc (Statistical Analysis); Lisa Buckingham BSc (Data Management) (McMaster University).

Adjudication: Vasco V. Mascarenhas MD, MBA, PhD (Hospital da Luz, Lisbon, Portugal).

Participating Clinical Sites

The following persons participated in or have Ethics Board approval to participate in the PREVIEW study at the time of submission of this

manuscript:

Canada

McMaster University Medical Centre (Hamilton, ON) - Olufemi R. Ayeni MD, PhD, FRCSC; Andrew Duong MSc; Ajay Shanmugaraj BSc; Nicole Simunovic MSc; Matthew Skelly BSc.

CHU de Québec-Université Laval (Quebec City, QC) - Etienne L. Belzile MD, FRCSC; Sylvie Turmel RN.

International

Erasmus University Medical Centre Rotterdam (Rotterdam, The Netherlands) Rintje Agricola MD, PhD.

Kyungpook National University Hospital (Daegu, South Korea) - Seung-Hoon Baek MD, PhD; Hoseok Lee MD, PhD; Ae-Sun Chang RN.

\section{Funding}

This study was funded by research grants from the Arthroscopy Association of North America, ConMed Research Grant, McMaster Surgical Associates, and the Canadian Institutes of Health Research (CEBA-223174).

\section{Availability of data and materials \\ Not applicable.}

\section{Ethics approval and consent to participate}

Ethical approval for this study was granted by the Hamilton Integrated Research Ethics Board (HIREB \#7829). Written informed consent will be obtained from participants and their parents/guardians.

\section{Consent for publication}

Not applicable.

\section{Competing interests}

Dr. Olufemi Ayeni is an Associate Editor of the BMC Musculoskeletal Disorders Journal.

Received: 24 October 2019 Accepted: 17 March 2020

Published online: 11 April 2020

\section{References}

1. Gosvig KK, Jacobsen S, Sonne-Holm S, Gebuhr P. The prevalence of camtype deformity of the hip joint: a survey of 4151 subjects of the Copenhagen osteoarthritis study. Acta Radiol. 2008;49(4):436-41.

2. Hack K, Di Primio G, Rakhra K, Beaule PE. Prevalence of cam-type femoroacetabular impingement morphology in asymptomatic volunteers. J Bone Joint Surg Am. 2010;92(14):2436-44.

3. Kapron AL, Anderson AE, Aoki SK, Phillips LG, Petron DJ, Toth R, et al. Radiographic prevalence of femoroacetabular impingement in collegiate football players: AAOS Exhibit Selection. J Bone Joint Surg Am. 2011;93(19): e111 (1-10).

4. Beck M, Kalhor M, Leunig M, Ganz R. Hip morphology influences the pattern of damage to the acetabular cartilage: femoroacetabular impingement as a cause of early osteoarthritis of the hip. J Bone Joint Surg (Br). 2005;87(7): $1012-8$.

5. Ganz R, Parvizi J, Beck M, Leunig M, Notzli H, Siebenrock KA. Femoroacetabular impingement: a cause for osteoarthritis of the hip. Clin Orthop Relat Res. 2003;(417):112-20. PMID:14646708. https://doi.org/10. 1097/01.blo.0000096804.78689.c2.

6. Philippon MJ, Patterson DC, Briggs KK. Hip arthroscopy and femoroacetabular impingement in the pediatric patient. J Pediatr Orthop. 2013;33(1):S126-30. 
7. Pathy $R$, Sink EL. Femoroacetabular impingement in children and adolescents. Curr Opin Pediatr. 2016;28(1):68-78.

8. Packer JD, Safran MR. The etiology of primary femoroacetabular impingement: genetics or acquired deformity? J Hip Preserv Surg. 2015;2(3): 249-57.

9. Agricola R, Heijboer MP, Ginai AZ, Roels P, Zadpoor AA, Verhaar JA, et al. A cam deformity is gradually acquired during skeletal maturation in adolescent and young male soccer players: a prospective study with minimum 2-year follow-up. Am J Sports Med. 2014;42(4):798-806.

10. Nepple JJ, Vigdorchik JM, Clohisy JC. What is the association between sports participation and the development of proximal femoral cam deformity? A systematic review and meta-analysis. Am J Sports Med. 2015;43(11):2833-40.

11. Siebenrock KA, Ferner F, Noble PC, Santore RF, Werlen S, Mamisch TC. The cam-type deformity of the proximal femur arises in childhood in response to vigorous sporting activity. Clin Orthop Relat Res. 2011;469(11):3229-40.

12. Leopold SS. Editor's spotlight/take 5: growth plate alteration precedes cam-type deformity in elite basketball players. Clin Orthop Relat Res. 2013;471(4):1081-3.

13. Frank JS, Gambacorta PL, Eisner EA. Hip pathology in the adolescent athlete J Am Acad Orthop Surg. 2013;21(11):665-74

14. Agricola R, Bessems JH, Ginai AZ, Heijboer MP, van der Heijden RA, Verhaar JA, et al. The development of cam-type deformity in adolescent and young male soccer players. Am J Sports Med. 2012;40(5):1099-106.

15. Johnson AC, Shaman MA, Ryan TG. Femoroacetabular impingement in former high-level youth soccer players. Am J Sports Med. 2012;40(6):1342-6.

16. Philippon MJ, Ho CP, Briggs KK, Stull J, LaPrade RF. Prevalence of increased alpha angles as a measure of cam-type femoroacetabular impingement in youth ice hockey players. Am J Sports Med. 2013;41(6):1357-62.

17. Siebenrock KA, Behning A, Mamisch TC, Schwab JM. Growth plate alteration precedes cam-type deformity in elite basketball players. Clin Orthop Relat Res. 2013;471(4):1084-91.

18. Feeley BT, Agel J, LaPrade RF. When is it too early for single sport specialization? Am J Sports Med. 2016:44(1):234-41.

19. Myer GD, Jayanthi N, Difiori JP, Faigenbaum AD, Kiefer AW, Logerstedt D, et al. Sport specialization, part I: does early sports specialization increase negative outcomes and reduce the opportunity for success in young athletes? Sports Health. 2015;7(5):437-42.

20. Myer GD, Jayanthi N, DiFiori JP, Faigenbaum AD, Kiefer AW, Logerstedt D, et al. Sports specialization, part II: alternative solutions to early sport specialization in youth athletes. Sports Health. 2016;8(1):65-73.

21. Kowalczuk M, Yeung M, Simunovic N, Ayeni OR. Does femoroacetabular impingement contribute to the development of hip osteoarthritis? A systematic review. Sports Med Arthrosc Rev. 2015;23(4):174-9.

22. Carsen S, Moroz PJ, Rakhra K, Ward LM, Dunlap H, Hay JA, et al. The Otto Aufranc award. On the etiology of the cam deformity: a cross-sectional pediatric MRI study. Clin Orthop Relat Res. 2014;472(2):430-6.

23. Eime RM, Harvey JT, Sawyer NA, Craike MJ, Symons CM, Payne WR. Changes in sport and physical activity participation for adolescent females: a longitudinal study. BMC Public Health. 2016;16:533.

24. Cadmus-Bertram L. Using fitness trackers in clinical research: what nurse practitioners need to know. J Nurse Pract. 2017;13(1):34-40

25. Taylor RW, Williams SM, Farmer VL, Taylor BJ. Changes in physical activity over time in young children: a longitudinal study using accelerometers. PLoS One. 2013;8(11):e81567.

26. Farooq MA, Parkinson KN, Adamson AJ, Pearce MS, Reilly JK, Hughes AR, et al. Timing of the decline in physical activity in childhood and adolescence: Gateshead millennium cohort study. Br J Sports Med. 2018; 52(15):1002-6

27. Gabel L, Macdonald HM, Nettlefold L, McKay HA. Physical activity, sedentary time, and bone strength from childhood to early adulthood: a mixed longitudinal HR-pQCT study. J Bone Miner Res. 2017;32(7):1525-36.

28. LaPrade RF, Agel J, Baker J, Brenner JS, Cordasco FA, Cote J, et al. AOSSM early sport specialization consensus statement. Orthop J Sports Med. 2016; 4(4):2325967116644241.

29. Jayanthi NA, LaBella CR, Fischer D, Pasulka J, Dugas LR. Sports-specialized intensive training and the risk of injury in young athletes: a clinical casecontrol study. Am J Sports Med. 2015;43(4):794-801.

30. Jayanthi N, Pinkham C, Dugas L, Patrick B, Labella C. Sports specialization in young athletes: evidence-based recommendations. Sports Health. 2013;5(3): 251-7.

31. Garber CE, Blissmer B, Deschenes MR, Franklin BA, Lamonte MJ, Lee IM, et al. American College of Sports Medicine position stand. Quantity and quality of exercise for developing and maintaining cardiorespiratory, musculoskeletal, and neuromotor fitness in apparently healthy adults: guidance for prescribing exercise. Med Sci Sports Exerc. 2011;43(7):1334-59.

32. Canadian Society for Exercise Physiology (CSEP). Canadian physical activity guidelines. 2019. https://csepguidelines.ca/. Accessed 20 Feb 2019.

33. DiFiori JP, Benjamin HJ, Brenner J, Gregory A, Jayanthi N, Landry GL, et al. Overuse injuries and burnout in youth sports: a position statement from the American medical Society for Sports Medicine. Clin J Sport Med. 2014;24(1):3-20.

34. Dollman J, Okely AD, Hardy L, Timperio A, Salmon J, Hills AP. A hitchhiker's guide to assessing young people's physical activity: deciding what method to use. J Sci Med Sport. 2009:12(5):518-25.

35. Price K, Bird SR, Lythgo N, Raj IS, Wong JY, Lynch C. Validation of the Fitbit one, Garmin Vivofit and jawbone UP activity tracker in estimation of energy expenditure during treadmill walking and running. J Med Eng Technol. 2017:41(3):208-15

36. El-Amrawy F, Nounou MI. Are currently available wearable devices for activity tracking and heart rate monitoring accurate, precise, and medically beneficial? Healthc Inform Res. 2015;21(4):315-20.

37. Simunek A, Dygryn J, Jakubec L, Neuls F, Fromel K, Welk GJ. Validity of Garmin Vivofit 1 and Garmin Vivofit 3 for school-based physical activity monitoring. Pediatr Exerc Sci. 2019;31(1):130-6.

38. Alsubheen SA, George AM, Baker A, Rohr LE, Basset FA. Accuracy of the vivofit activity tracker. J Med Eng Technol. 2016;40(6):298-306.

39. Butte NF, Ekelund U, Westerterp KR. Assessing physical activity using wearable monitors: measures of physical activity. Med Sci Sports Exerc. 2012:44(1 Suppl 1):S5-12.

40. Eston RG, Rowlands AV, Ingledew DK. Validity of heart rate, pedometry, and accelerometry for predicting the energy cost of children's activities. J Appl Physiol. 1998;84(1):362-71.

41. Smolders JMH, Speirs AD, Frei H, Beaule PE. Higher patient activity level and subchondral stiffening in asymptomatic cam femoroacetabular impingement subjects. J Hip Preserv Surg. 2018;5(3):259-66.

42. Schenker ML, Martin R, Weiland DE, Philippon MJ. Current trends in hip arthroscopy: a review of injury diagnosis, techniques and outcome scoring. Curr Opin Orthop. 2005;16:89-94.

43. Varni JW. The PedsQL: measurement model for the pediatric quality of life inventory. 2016. http://wwwpedsql.org. Accessed 11 Sep 2019.

44. Li AE, Jawetz ST, Greditzer HG 4th, Burge AJ, Nawabi DH, Potter HG. MRI for the preoperative evaluation of femoroacetabular impingement. Insights Imaging. 2016;7(2):187-98.

45. Cotorro AR, Philippon M, Briggs K, Boykin R, Dominguez D. Hip screening in elite youth tennis players. Abstract 60. Monaco: IOC World Conference on Prevention of Injury and IIIness in Sport; 2014.

46. Kuhlman GS, Domb BG. Hip impingement: identifying and treating a common cause of hip pain. Am Fam Physician. 2009:80(12):1429-34.

47. Nötzli HP, Wyss TF, Stoecklin CH, Schmid MR, Treiber K, Hodler J. The contour of the femoral head-neck junction as a predictor for the risk of anterior impingement. J Bone Joint Surg (Br). 2002;84(4):556-60.

48. Durant RH, Baranowski T, Davis H, Thompson WO, Puhl J, Greaves KA, et al. Reliability and variability of heart rate monitoring in 3-, 4-, or 5-yr-old children. Med Sci Sports Exerc. 1992;24(2):265-71.

49. Hullmann SE, Ryan JL, Ramsey RR, Chaney JM, Mullins LL. Measures of general pediatric quality of life: Child Health Questionnaire (CHQ), DISABKIDS Chronic Generic Measure (DCGM), KINDL-R, Pediatric Quality of Life Inventory (PedsQL) 4.0 Generic Core Scales, and Quality of My Life Questionnaire (QoML). Arthritis Care Res. 2011;63(Suppl 11):S420-30.

\section{Publisher's Note}

Springer Nature remains neutral with regard to jurisdictional claims in published maps and institutional affiliations. 